\title{
Impact Study and Evaluation of Higher Modulation Schemes on Physical Layer of Upcoming Wireless Mobile Networks
}

\author{
Heba Haboobi ${ }^{1}$, Mohammad R Kadhum ${ }^{2}$ \\ Faculty of Arts, Science \& Technology \\ University of Northampton \\ Northampton, U.K.
}

\begin{abstract}
In this paper, the higher modulation formats (128 and 256) Quadrature Amplitude Modulation (QAM), for modulation/demodulation the digital signal of the currently used Orthogonal Frequency Division Multiplexing (OFDM) system, is proposed, explored and evaluated at a wireless transmission system. The proposed modulation schemes are utilized to study the impact of adding extra bits for each transmitted sample on system performance in terms of the channel capacity, Bit Error Rate (BER) and Signal to Noise Ratio (SNR). As such, the key purpose of this research is to identify the advantages and disadvantages of using higher modulation schemes on the physical layer (PHY) of future mobile networks. In addition, the trade-off relation between the achieved bit rate and the required power of the receiver is examined in the presence of the Additive White Gaussian Noise (AWGN) and Rayleigh noise channels. Besides, the currently employed waveform (OFDM) is considered herein as an essential environment to test the effect of receiving additional complex numbers on the constellations table. Thus, investigate the ability to recognize both the phase and amplitude of intended constellations for the upcoming design of wireless transceivers. Moreover, a MATLAB simulation is employed to evaluate the proposed system mathematically and physically in an electrical back-to-back transmission system.
\end{abstract}

Keywords-Orthogonal Frequency Division Multiplexing (OFDM); Quadrature Amplitude Modulation (QAM); Bit Error Rate (BER); Signal to Noise Ratio (SNR); Bandwidth (BW); Additive White Gaussian Noise (AWGN); Rayleigh noise; physical layer (PHY)

\section{INTRODUCTION}

Enlarged growth of propagated data over wireless mobile networks is associated directly with an expanded range of emerging data-hungry applications. Hence, nowadays, using smart phones, users are looking for downloading and uploading huge amounts of data rather than the conventional operations. Thus, the predicted scenarios for the next generation of mobile are considered as an essential driving force for introducing higher modulation schemes [1].

Recently, many research studies have proposed new solutions to meet with the future market demand for mobile networks [2]. Consequently, achieve developed rates of channel capacity at acceptable limits of errors get overwhelming interest in terms of R\&D. As such, high efforts have been made to promote both bit rate and bandwidth (BW) efficiency by employing higher modulation formats like 64 Quadrature Amplitude Modulation (QAM).
Nevertheless, so far, investigation in more advanced formats of modulation like the 128 and 256 QAM is yet not introduced for future wireless networks of mobile [3].

It's worth noting that, comparing to the lower modulation formats, the higher order schemes can significantly improve both BW efficiency and maximum bit rate with raising the minimum limits of the Signal to Noise Ratio (SNR) to keep the required signal at the acceptable level of the Bit Error Rate (BER). As such, for the wireless transmission system, the modulation and demodulation process can play a key role in regulating the BER levels and maximum rates of transmitted data [4].

The big challenge for future wireless networks is increasing transmission and reception rates for mobile communications and decreasing required levels of power and BER [5]. That's mean, the required modulation format is essentially selected in accordance with the future system performance, in terms of the bit rate, and the BER.

Utilizing an appropriate type of a modulation format for the advanced mobile transmission is critical due to key technical limitations. Thus, factors like an optimal usage of the BW, limitation of received power and probability of constellation table noise are major restrictions for developers in the modulation format field of wireless communication.

From digital signal processing (DSP) perspective, advanced digital modulation schemes can play a big role in expanding channel capacity by transmitting high amounts of data per each slice of time and with an acceptable immunity of noise particularly when the required level of power is afforded [6]. Thus, the performance in terms of maximum data bit rate and minimum error rate of a modulation scheme is decided depending on the efficient usage of the BW and power.

The key question of this study is, can the higher modulation formats be a good solution for a developed performance of future wireless mobile technology?

In this context, this study discusses how the developed modulation formats can impact the performance of transmission in a thorough manner by comparing not only the major principles of the conventional modulation formats but also including the most recent one. As such, investigating a higher scheme like 128 and 256 QAM to get a promising progress in this field of research. Hence, emphasis on the trade-off relation 
between the improved BW efficiency and required level of received power for future mobile networks.

Depending on Shannon, the channel capacity can be decided by the maximum number of transmitted bits throw an obtainable BW and at an acceptable level of errors. Thus, in digital wireless communication, the quality of the transmission service is typically measured by both the BER and the maximum achieved bit rate [7].

The main principle beyond designing an optimal digital communication system is to obtain lower levels of error and get more efficient usage of channel BW simultaneously. Hence, an efficient modulation system can basically assistant a better spectral efficiency (SE), where the SE represents a number of bits transmitted per second for each Hertz of the BW [8].

To accommodate the developed schemes of modulation format in the physical layer (PHY) of wireless mobile networks, the current waveform Orthogonal Frequency Division Multiplexing (OFDM) is utilized due to its high resistance against the Inter-Symbol Interference (ISI), better BW efficiency, and improved bit rate of transmission [9]. Thus, the newly examined formats of modulation (128 QAM \& 256 QAM) are explored employing the most popular transmission air interface OFDM.

Since the orthogonality is the key feature of the currently applied waveform, a better level of transmission can be delivered by the OFDM in comparison with the old-style Frequency Division Multiplexing (FDM) [10].

It's worth noting that, the impact of unwanted signals (noise) is considered herein for both the line of sight (LoS) and non-line of sight (NLoS) transmission. Thus, different models of channels like the Additive white Gaussian noise (AWGN) and Rayleigh noise are adopted to compare the performance of the transmission system for the most popular modulation schemes.

The rest of the paper is structured as follows: Section 2 demonstrates a literature review of the prepared study. Section 3 deliberates theoretically the main concepts of the proposed system highlighting physically and mathematically the fundamentals beyond it. Section 4 simulates numerically system performance (channel capacity and BER). Section 5 concludes the outlines of the paper.

\section{Literature REVIEW}

Since the main target of a future wireless system designer is to transmit bigger data rate within the offered $\mathrm{BW}$ and at an affordable expense [11], digital Modulation still has a high impact relation on the world of the developed throughput communication system. In this context, various studies about the relation between the most common modulation formats and channel models of the wireless communication system are investigated.

Regarding the Binary Phase Shift Keying (BPSK), it's the simplest form of modulation formats which is used for representing digital data employing two changed phases of each available subcarrier. The two utilized phases are detached by 180 degree, and a regular angular interval around a circle is ordinarily applied to place the selected points at the constellation table.
The best immunity to distortion is achieved due to the maximum separation in phase between adjacent constellation points. Thus, two phases are assigned arbitrarily, $(0$ and $\pi)$, representing the binary " 0 " and " 1 ".

Besides, their circular position makes them able to get an equal energy of transmission. In addition, the complex numbers represented by this way will have a similar modulus due to apply the same real and imaginary part for each point [12].

Regarding the Quadrature Phase Shift Keying (QPSK), it is another form of Phase Shift Keying where couple bits are utilized for each used subcarrier. Hence, employing the same $\mathrm{BW}$, one of four phase shifts $(0,90,180$, or 270$)$ degree are possibly selected to represent the generated points.

In comparative to the BPSK scheme, this modulation format is used to get a dual bit rate for a similar maintaining boundary of BW. Nevertheless, to achieve the same BPSK level of the BER, the QPSK need to twice the received power due to transmitting two bits simultaneously.

The investigations about employing the digital modulation with the OFDM particularly low modulation schemes like BPSK, QPSK were started in last three decades. The performance of the OFDM based BPSK, QPSK, etc., was explored in terms of the BER and channel capacity. Hence, a number of studies discussed in detail the low bit rate transmission and showed the trade-off relation between the size of achieved capacity and the SNR, particularly, a power of the signal and how it improved to recover the overall signal in the receiver.

In addition, the effect of transmission through noisy channels has been shown, thus, the performance (BER, bit rate) for a transmitted signal has been investigated under the AWGN channel response showing how the added noise can eliminate the performance of transmission for gained capacities at intended limits of errors.

Moreover, the influence of the AWGN is experimented for different received power at the receiver side demonstrating the impact of the applied power on the received signal. Furthermore, some researchers investigated the effect of expanding the available BW and how that can improve the bit rate for low modulation format [13].

However, those studies discussed the transmission performance of both BER and channel capacity for only low bit rate modulation format, thus, explore the activity for low capacity systems.

It's worth noting that, whenever the wanted bit rate is increased (16 PSK or more), it is strongly advisable to move to the Quadrature Amplitude Modulation (QAM) due to larger distances among the adjacent points in the constellation table. On the other hand, detect the points with both the phase and modulus due to having different amplitudes rises the involved complexity of the demodulation system.

From a general perspective, the QAM is a modulation mechanism, in which, both the amplitude and phase are utilized to express the modulated points for each frequency subcarrier.

The combination of shifted amplitude and phase produces higher modulation system with enhanced data representation. Hence, the mixture of changing phases and amplitudes can improve data transmission efficiency. 
In addition, such a kind of modulation can supply a higher bit rate than formerly mentioned modulation scheme (PSK) for digital communications of mobile. Thus, increasing the number of the indicated points at the constellation table.

Besides, a square grid arrangement with equivalent horizontal and vertical spacing is applied for representing the QAM points, where the amplitude can vary with the phase at the Inphase (I) and Quadrate-phase (Q) plane.

The possibility of transmitting further bits per symbol is achieved employing orderly higher modulation schemes. Hence, supply room for added points inside the constellation table, to raise the ability of transmitting a similar amount of data in a smaller BW.

However, this is come up with raising the level of noise since constellation points number is enlarged and the spaces among them are narrowed resulting in higher BER at the constellation table.

This, nevertheless, can improve the BW efficiency but with less reliability in comparative with the lower order of modulation formats. However, this more liable to noise system is treated by promoting the needed power of the signal at the receiver side.

Regarding the first most popular one of the QAM family, which is termed as the 16 QAM, number of studies started to appear exploring an alternative approach for the digital modulation depends on both phase and amplitude of the signal instead of the phase only.

The new style of the modulation represented a big movement in a wireless digital modulation due to its ability to improve BW efficiency. Hence, employ an extra number of bits per each sample (4 bits) to generate each complex number. The performance in terms of the BER and channel capacity for the OFDM based 16 QAM, were discussed extremely by a number of researchers.

In addition, the close relation between the SNR and BER investigated to show the effect of adding extra bit for each sample in the constellation table. Hence, the received electrical power was also another determination for the improved signal.

As a result, the received signal is not recovered perfectly unless increase the power at the receiver side. The utilized BW was increased side by side by upgrading the modulation format giving an extra improvement for the performance in terms of the maximum bit rate and the BER of system.

Moreover, the effect of testing the AWGN channel has been demonstrated showing how the performance of channel capacity for transmitted signal was decreased due to increased BER [14]. However, this study demonstrated the system performance for only 16 QAM and future market demands requires more and more capacity like 32, 64, 128, 256.

Recently, researchers investigated another developed modulation scheme for upgrading the digital wireless transmission systems. This investigation showed that by increasing the number of bits for each transmitted sample (5 bits), the performance in terms of capacity and BER for the OFDM based 32 QAM was raised.
Nevertheless, this improvement caused in increasing the required SNR to mitigate the internal constellation noise.

In addition, the influence of the unwanted signal (AWGN) was explored demonstrating the relationship between increased SNR and reduced BER due to a promoted power of the signal. Hence, the utilized power for the received signal was strengthened against the unwanted power of the noise.

Moreover, a number of the exercised received powers was examined clarifying how the SNR is improved side by side with the raised power at the receiver side.

Improving the level of modulation format was accompanied by a number of trials for expansion the utilized BW and get some extra enhancements for the performance of wireless transmission systems with indeed an added cost [15].

However, the hangry data applications still looking for employing further developed schemes to sustain high capacities at a good level of errors.

Very recent, new studies have demonstrated the influence of utilizing a modern developed modulation format (64 QAM). This advanced modulation which is considered lately by the Long-Term Evolution (LTE) can accommodate 6 bits per each sample. Thus, improve the spectrum efficiency of the employed BW.

In addition, researchers have focused their attention on the trade-off relation between the enhanced performance of both maximum bit rate and the BER, and the expanded limits of power for a received signal. Hence, clarify the ability of the developed system to address the generated noise due to raised interference among the intended values of the signal based the new modulation format.

On the other hand, the impact of the uniform AWGN in a wireless channel showed how the developed system needs an extra power to mitigate the signal at the receiver side.

Besides, different values of received powers have been tested exploring how the optimal value of the SNR was achieved [16].

The promoted modulation schemes synchronized with indicated efforts to invest in the obtainable BW. Hence, seeking further solutions to enhance the efficiency of spectrum for acceptable boundaries of errors.

Nevertheless, the need for a higher data rate of greedy data services is still required by the future generation of wireless networks.

In a wireless mobile radio channel, the type of attenuation for a transmitted signal is decided by the nature of the propagated signal and the features of the utilized channel. Hence, various transmitted signals will suffer from various kinds of fading effects due to the relation between the parameters of both the channel like delay/doppler and the signal like symbol interval.

To explore the underlying behaviour of a wireless communications system in the absence of nonlinearity factors of noise, the AWGN model is employed. Hence, diving the internal behaviour of the spread signal before considering the other complicated phenomena like interference, dispersion, etc. 
This basic, tractable and organized mathematical model added a steady spectral density linearly which is represented as watts per Hertz of the available BW over a specific transmission media.

In addition, the white noise with a Gaussian distribution of modulus is assumed for a flat channel with a fixed transmitter and receiver [17].

To illustrate the NLoS effect of radio signal propagation between the transmitter and receiver, the Rayleigh channel is discussed.

Such statistical model is important for signals which basically suffer from a similar attenuation, but different phases of arrival. Hence, for a wireless signal passed through the communication channel, the changed amplitudes of a signal for various coming times are assumed according to the Rayleigh distribution. Thus, the Rayleigh model is mainly applied due to the multipath phenomenon at the receiver [17].

To explain more about the multipath propagation, in mobile communications, the multipath phenomenon occurs when a radio signal is received at antenna by more than one path. This, as a result, influences the quality of telecommunications due to shifted phases of the same signal.

It's worth noting that, a maximum bit rate is directly related to the intended level of the BER. Hence, the BER considers as a significant parameter in assessing the performance of the digital wireless transmission system. Thus, the BER is utilized to examine the overall performance of the electrical back-toback transmission system (transmitter, receiver and wireless channel) referring to the rate of error occurrence for a delivered signal.

\section{SySTEM MODEL}

As is seen in Fig. 1, utilizing the OFDM environment, the digital signal is converted as the first step to its frequency domain using the proposed system of higher modulation formats (128 and 256 QAM), thereafter, the signal is transformed to the time domain using Inverse Fast Fourier Transform (IFFT).

The time guard interval, Cyclic Prefix (CP) is added at this stage of transmission to supply an appropriate level of protection for the transmitted symbols. Hence, these offered intervals of time play a big role in preventing any probable interference between transmitted symbols. Thus, sustain the

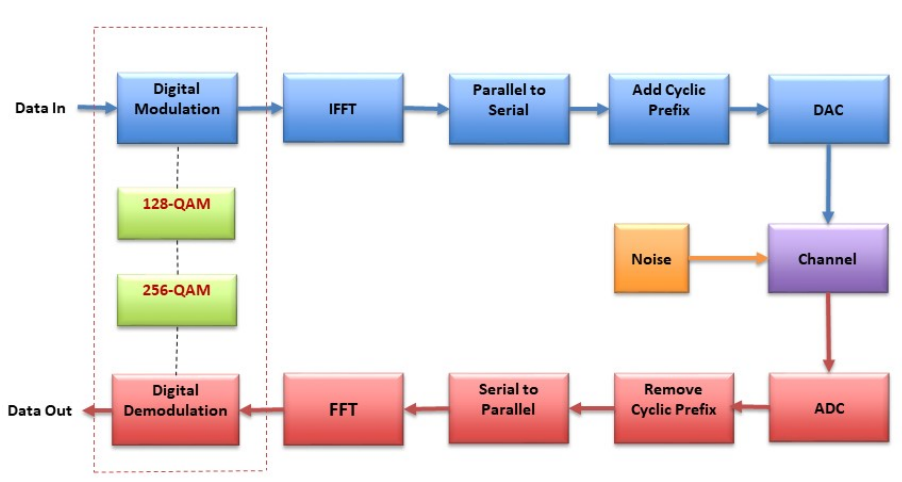

Fig. 1. Proposed OFDM transceiver including the higher modulation formats. robustness of the transmission system against a probably happened Inter-Symbol Interference (ISI).

Utilizing a proper sampling frequency digital to analog converter $\left(F_{D A C}\right)$, the prepared signal is converted to analog domain to be broadcasting later by an antenna.

In the receiver side, inverse processing is applied. The wireless received signal is converted back to its digital domain using a corresponding sampler analog to digital converter $\left(F_{A D C}\right)$. The CP's that followed symbols of the OFDM are removed periodically to conclude the main signal in the time domain.

Employing Fast Fourier Transform (FFT), the received samples are turned back into the frequency domain. Finally, the digital values are recovered from their complex numbers using the demodulation operation.

The main concept beyond the OFDM is to divide a signal stream with high data rate into a group of low data rate which are simultaneously transmitted through a group of subcarriers.

As the modulation / demodulation process represents a key stage of the OFDM system, it is quite important to discuss, from a mathematical perspective, some related concepts that can affect directly the transmission operation.

In the transmitter side, particularly, in the modulation process, each token of the binary digits is converted to a complex number which is expressed in Cartesian forms as follow [18]:

$$
C_{k}=I_{k}+j Q_{k}
$$

where $j=\sqrt{-1}$, and $I, Q$ represents the real and imaginary parts for $k^{t h}$ complex number respectively.

In this context, the mathematical relationship between the amplitude (A) and a produced complex number is clarified as follow [19]:

$$
A_{k}=\sqrt{I_{k}^{2}+Q_{k}^{2}}
$$

In addition, the relationship between the phase $(\Theta)$ and a complex number is illustrated as follows [18]:

$$
\Theta_{k}=\arctan \left(\frac{Q_{k}}{I_{k}}\right)
$$

Thus, both the amplitude and phase are concluded based on the complex number formula in the Cartesian domain. This is, however, not considered for all mathematical operation of the complex number. Hence, the Cartesian form can fit for addition and subtraction operations more than multiplication and division.

To address this issue, the Polar form is accounted to state both multiplication and division in an easier way. The complex number formula in the Polar domain is demonstrated as follows [18]:

$$
C=A e^{j \Theta}
$$

Then, according to Euler's formula [18]: 


$$
e^{j \Theta}=\cos \Theta+j \sin \Theta
$$

This, as result, leads to clarify a complex number in a sinusoidal form as follows [18]:

$$
C=A \cos \Theta+A j \sin \Theta
$$

Consequently, $I$ (real part) $=A \cos \Theta$ and $Q$ (imaginary part $)=A \sin \Theta$.

In the receiver side, particularly, when the channel response is counted, it is very necessary to make use of channel estimation concepts due to probably higher changes in both phase and amplitude of the transmitted signal over a wireless channel.

Thus, there is no benefit of applying the demodulation process, before achieving such a kind of corrections. According to this principle, the pilot-aided scheme with two key steps is applied to perform the equalization for the received signal as follows:

The first step is calculating the inverse of the channel response to mitigate the bad effect of the channel. Then, averaging the received pilots over $m$ transmitted samples to reduce the noise of signal and then to recover the signal itself.

To perform the equalization in a simple way, the Channel Transfer Function (CTF) is estimated. This, however, needs to make both the amplitude and phase of each pilot sample are known. Hence, the training samples $T_{k}$ are transmitted periodically with recognized magnitude and phase as the following [19]:

$$
T_{k}=X_{k} * e^{j \phi_{K}}
$$

where $X_{k}$, and $\left(\phi_{K}\right)$ represent the amplitude and phase of the $K_{t h}$ transmitted sample, respectively.

The equivalent received training samples $R_{k}$ is [16]:

$$
R_{k}=Y_{k} * e^{j \varphi_{K}}+N_{k}
$$

where $Y_{k}$, and $\left(\varphi_{K}\right)$ represent the amplitude and phase of the $K_{t h}$ received sample, respectively and $N_{k}$ is the $K_{t h}$ sample noise.

By making use of the identified transmitted and received training samples, the CTF in frequency domain $E_{k}$, is determined as the following [19]:

$$
E_{k}=\frac{R_{k}-N_{k}}{T_{k}}
$$

Thus, the estimated CTF can be explained as the following:

$$
\hat{E}_{k}=E_{k}+\frac{N_{k}}{T_{k}}
$$

Finally, the other received complex values in frequency domain $V_{i}$ is equalized by multiplying the inverse of the estimated CTF, $E_{k}^{-1}$ as follow [19]:

$$
\breve{V}=\hat{E}_{k}^{-1} * \dot{V}_{i}
$$

Regarding the probable shapes of complex numbers on the constellation table, the following equation achieves this purpose [18]:

$$
Y=2^{X}
$$

where $Y$ represents the total number of probabilities which might be assigned for each sample employing $X$ of input bits.

Despite each spectrum of subcarrier can coincide with the others, the ability to extract each subcarrier is achieved over the digital signal processing. Hence, this overlapped property of subcarriers increases the spectrum efficiency of the current OFDM in comparative with the previous multicarrier design of waveform.

Thus, the OFDM technique splits a wireless channel into smaller subcarriers each one is modulated with an amount of data according to the applied modulation format. The improved efficiency of the OFDM spectrum is gained due to applying the orthogonality between adjacent subcarriers. Consequently, obtain a larger benefit for the same offered BW.

According to Shannon's theorem, the channel capacity represents the maximum achieved bit rate with a vanished amount of errors as follows [20]:

$$
\text { Capacity }=B W \cdot \log _{2}(1+S N R)
$$

Besides, the SNR is gained as follow:

$$
S N R=P_{\text {receiver }} / P_{\text {noise }}
$$

As such, to improve the obtained channel capacity, it's better to make an extra investigation in the field of $\mathrm{BW}$ efficiency than going with expanding the offered BW itself since such expanded resources require a highly increased cost.

In this study, the modulation operation is explored in the presence of AWGN and Rayleigh channels. Hence, the received signal $R$ is composed of the transmitted signal $T$ multiplied by the response of the channel $E$, where $E=1$ with the AWGN and $E \neq 1$ with the Rayleigh. Furthermore, the composition is combined with the AWGN, which is represented here as $N$, as follows [21]:

$$
R=E T+N
$$

It's worth noting that, the assigned length $k$ of the utilized IFFT/FFT represents the total number of subcarriers presented in the system. This is, nevertheless, not actually account for a real number of the employed subcarriers. Thus, when the system is supported with $k$ subcarriers, the utilized subcarriers for data transmission is about half of them. This is due to apply an equivalent number of conjugates which are employed in converting the signal from the frequency domain to the time domain. 
TABLE I. KEy PARAMETERS OF OFDM SySTEM

\begin{tabular}{|c|c|}
\hline Parameter & Value \\
\hline FFT size & 40 \\
\hline Sampling frequency & $40 \mathrm{MHz}$ \\
\hline Number of subcarriers & 15 \\
\hline Cyclic prefix & 0.25 \\
\hline
\end{tabular}

In addition, a specific duration is assigned for the $\mathrm{CP}$ to ensure more reliable transmission system.

\section{EXPERIMENTAL RESULTS}

In this part, numerical simulation with the MATLAB is introduced for the promoted transmission system demonstrating the advantages and disadvantages of adding higher modulation schemes for future mobile networks. Thus, examining experimentally the performance in terms of a maximum bit rate and the BER for the OFDM based 128 QAM and 256 QAM to explore the behaviour of wireless channel responses over variant modulation schemes (low and high).

The experiment is set up herein for 15 frequencies of subcarriers where the advanced modulation formats are considered side by side with the conventional modulation schemes. Besides, as the modulation system is the core of this proposed system, the number of stated bits for each used subcarrier is accurately fixed in accordance with the corresponding level of employed modulation schemes. The optimal parameters of the configured model that utilized for a wireless electrical back-toback system is basically achieved under the conditions shown in Table I.

As is shown in Fig. 2, the newly proposed modulation formats, 128, and 256 QAM can improve the transmission bit rate compared to currently applied, 64 QAM, to about $16 \%$ and $33 \%$, respectively.

In addition, in comparison with the 32 QAM and 16 QAM, the higher modulation 256 QAM increase the overall channel capacity by about $60 \%$ and $100 \%$ sequentially.

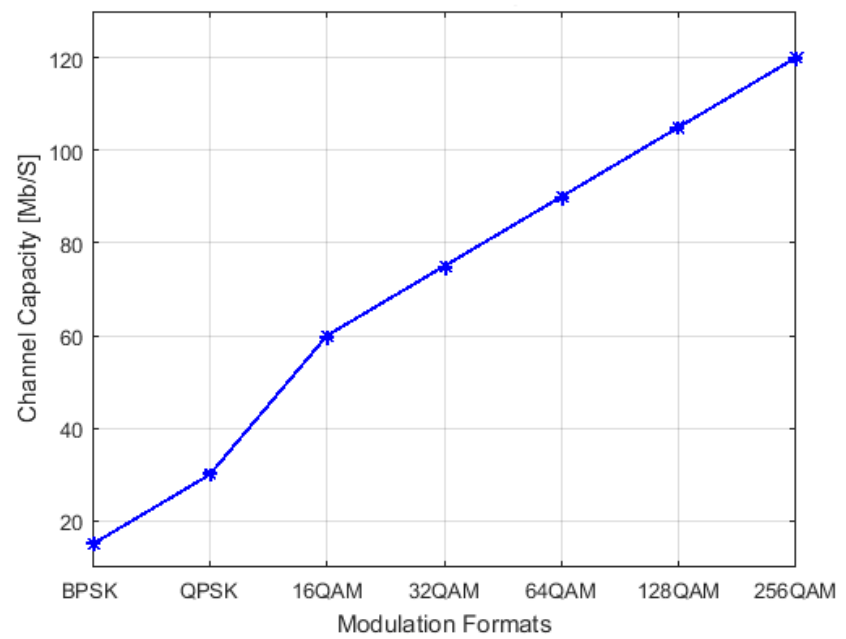

Fig. 2. Maximum achieved bit rate of different modulation formats including the 128 and 256 QAM
Moreover, 3- and 7-times enhancement are registered for the higher modulation (256 QAM) in comparison to both QPSK and BPSK.

This, however, comes up with raising the level of errors for transmitted samples due to enlarged interference at the constellation map. As such, a higher BER is presented for each utilized subcarrier because of the need for higher received power to sustain the required limits of errors at $10^{-3}$.

The experiment illustrates also, how the BER of the employed subcarriers is varied for diverse schemes of modulation under a similar level of the SNR and over the AWGN channel.

As is clear in Fig. 3, the BERs of the applied subcarriers are calculated for the currently employed modulation format (64 QAM). The measured BER is essentially achieved with an appropriate level of the SNR, which is equivalent herein to $23 \mathrm{~dB}$. Accordingly, the acceptable limits of errors are gained due to supplying a suitable received power.

This scenario, however, is not typically fit for higher formats of modulation like 128 and 256 QAM.

According to Fig. 4, utilizing the same level of the SNR, the BER gets worse with increasing the modulation technique to the 128 QAM.

Furthermore, as is shown in Fig. 5, an extra rise of the BER level is recorded with moving to the higher modulation format (256 QAM) and keep the SNR at $23 \mathrm{~dB}$. This fundamentally, due to reduce the distances between the adjacent samples on the constellation table resulting in inability in recognizing the received signal of the enlarged modulation schemes.

To explain the impact of increasing the supplied levels of received power on the signal strength, the SNR is raised, firstly, by about $3 \mathrm{~dB}$ to be more suitable for the $128 \mathrm{QAM}$.

As is clarified in Fig. 6, the new drawn map of the BERs refers to a feasible enhancement at the gained averages of errors with 128 QAM due to reduce the influence of interference among the constellations.

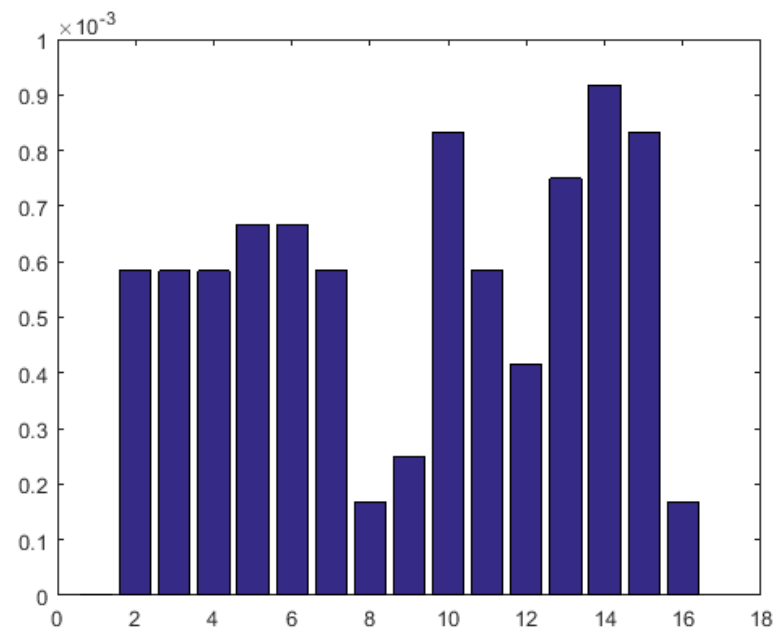

Fig. 3. BERs' map of utilized subcarriers with 64 QAM and $S N R=23$. 


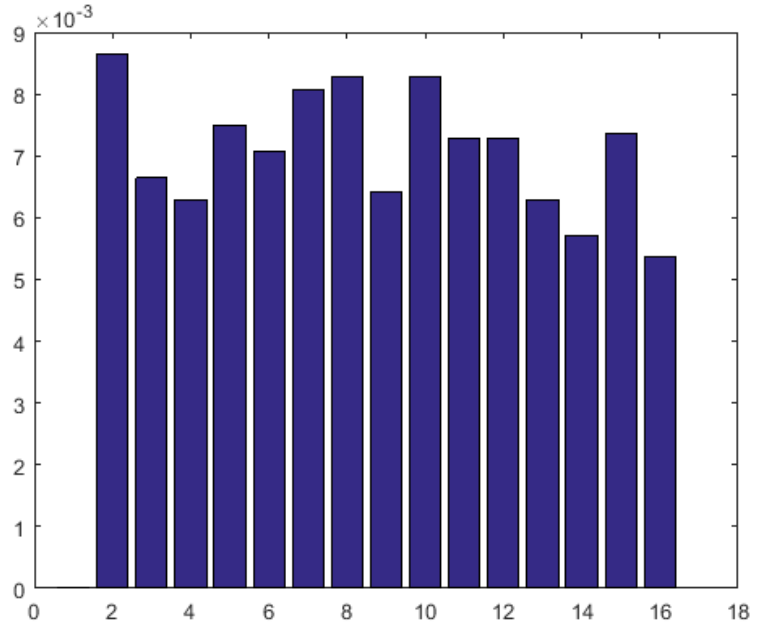

Fig. 4. BERs' map of utilized subcarriers with 128 QAM and SNR=23.

This level of the SNR, as is shown in Fig. 7 is, however, still not able to support the accepted limit of the BER for the 256 QAM, since the improved power yet less than the induced interference among the samples at the receiver side.

Consequently, to obtain an acceptable amount of errors rate, as is seen in Fig. 8, the SNR is further expanded to about $29 \mathrm{~dB}$, hence, an extra $3 \mathrm{~dB}$ improvement is realized for the employed SNR to sustain the BER limits of applied subcarriers with the higher order modulation (256 QAM).

For more details, the relationship between the overall calculated BER and the set level of the SNR is investigated for variant modulation schemes and over different channel models (AWGN, Rayleigh).

As is clear in Fig. 9, the performance in terms of the SNR and BER is firstly examined for the presently utilized 64 QAM and in presence of both the AWGN and Rayleigh noise channels.

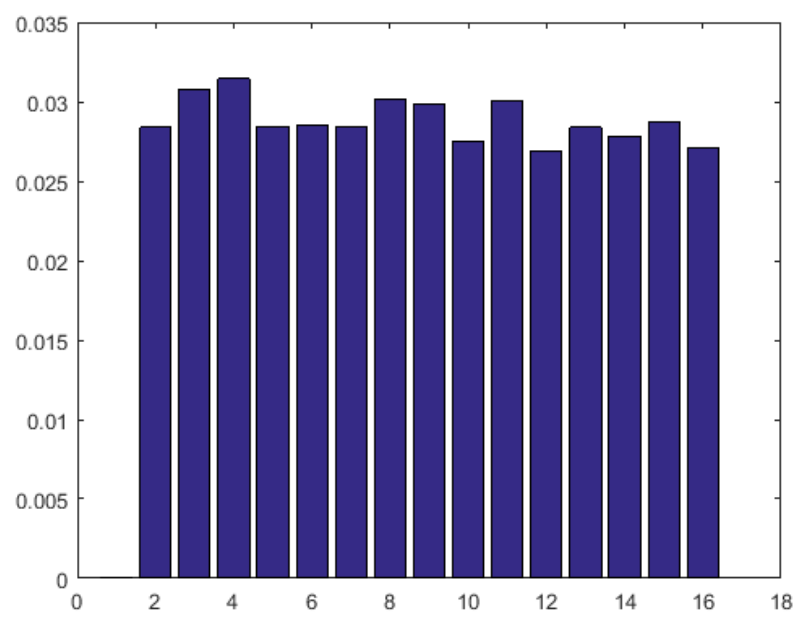

Fig. 5. BERs' map of utilized subcarriers with 256 QAM, SNR=23.

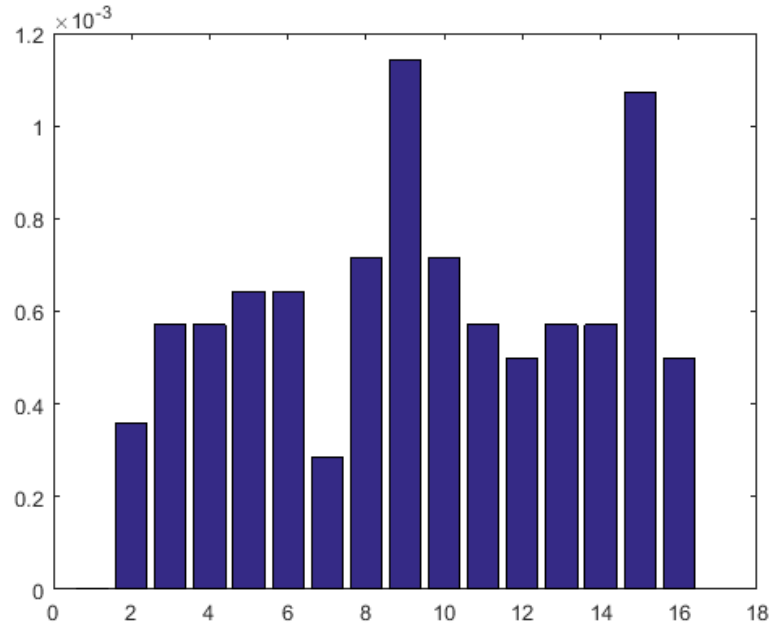

Fig. 6. BERs' map of utilized subcarriers with 128 QAM, SNR=26.

It's, also, noticed that the obtained results of the 64 QAM modulation format are varied depending on the channel model, hence, the electrical signal is received over the AWGN with a lower level of power in comparison with the Rayleigh channel. Thus, the required limit of SNR is increased with the Rayleigh due to the impact of channel response on the transmitted signal.

Regarding the primarily proposed modulation formats (128 QAM), it is observed in Fig. 10, that agreeable limit of the BER calculated for the 128 QAM is achieved with a higher level of the SNR. This basically results from the reception system attempt to recover the signal in good condition. Thus, over the both considered channels, the BER is improved with increasing the SNR due to mitigating the influence of convergent samples at the constellation table.

In addition, the dynamic range of the utilized SNR shows a $3 \mathrm{~dB}$ difference between the minimum limits of received power for both the currently employed 64 QAM and the proposed 128 QAM.

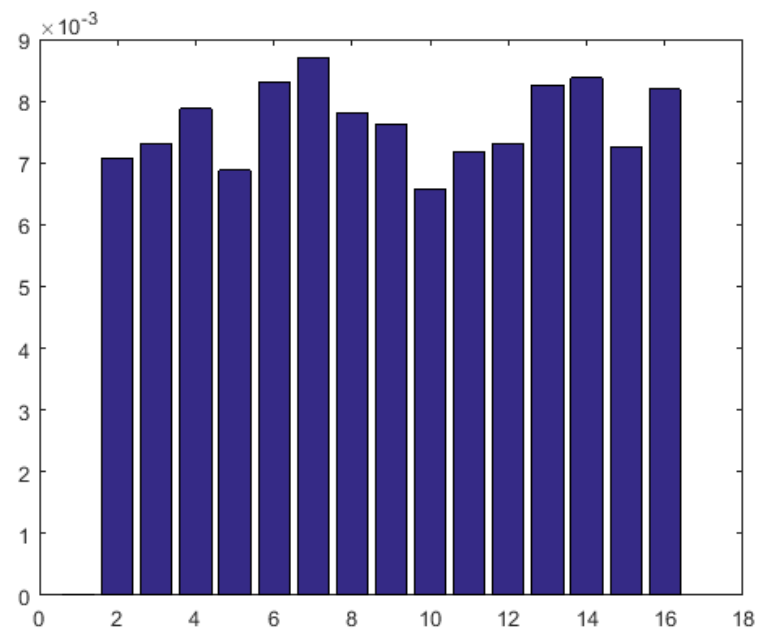

Fig. 7. BERs' map of utilized subcarriers with 256 QAM, SNR=26. 


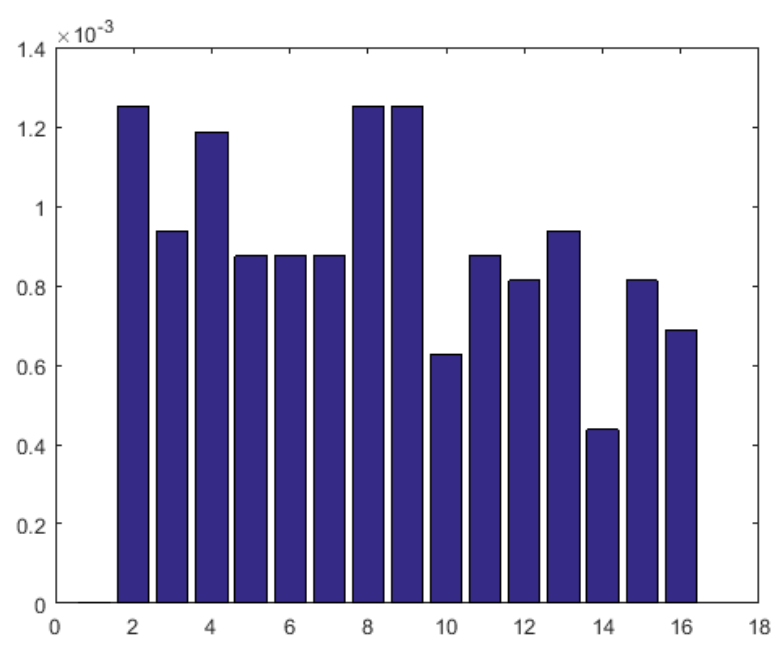

Fig. 8. BERs' map of utilized subcarriers with 256 QAM, SNR=29.

Regarding the secondly proposed higher order modulation scheme (256 QAM), as is obvious in Fig. 11, the intended limit of receiving power is further increased with promoting the modulation to a better degree of transmission, hence, an extra larger level of the SNR is recorded with the typically acceptable average of error.

Besides, for both adopted channels (AWGN and Rayleigh), whenever, the supplied levels of the SNR are raised, the BER limits are enhanced accordingly. This is fundamentally occurred since the impact of constellations interference is significantly reduced at the receiver side.

It's worth noting that the dynamic range of the applied SNR is even more affected, where about $6 \mathrm{~dB}$ variance is registered between the actually used modulation scheme (64 QAM) and the herein proposed (256 QAM).

As is seen in Fig. 12, the trade-off relation between the

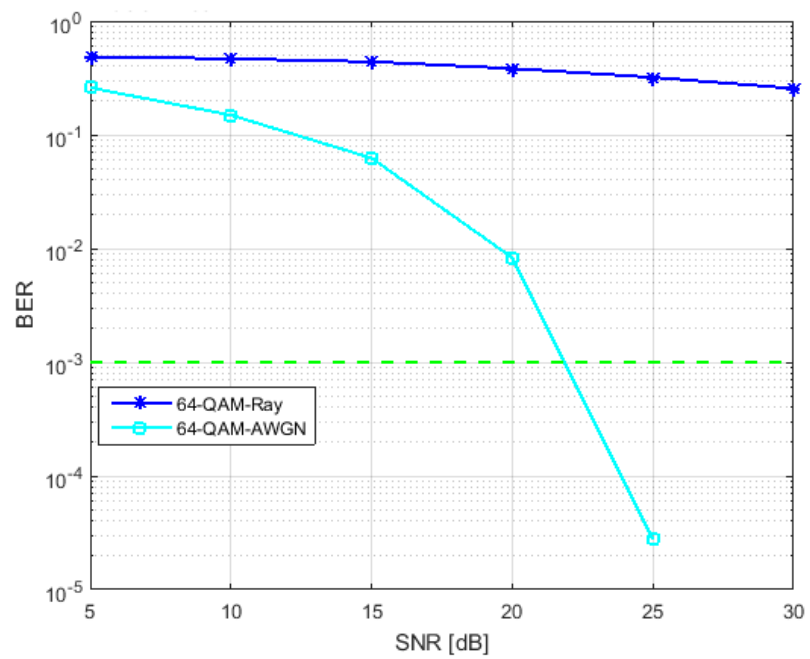

Fig. 9. Relation between BER and SNR for 64 QAM over AWGN and Rayleigh.

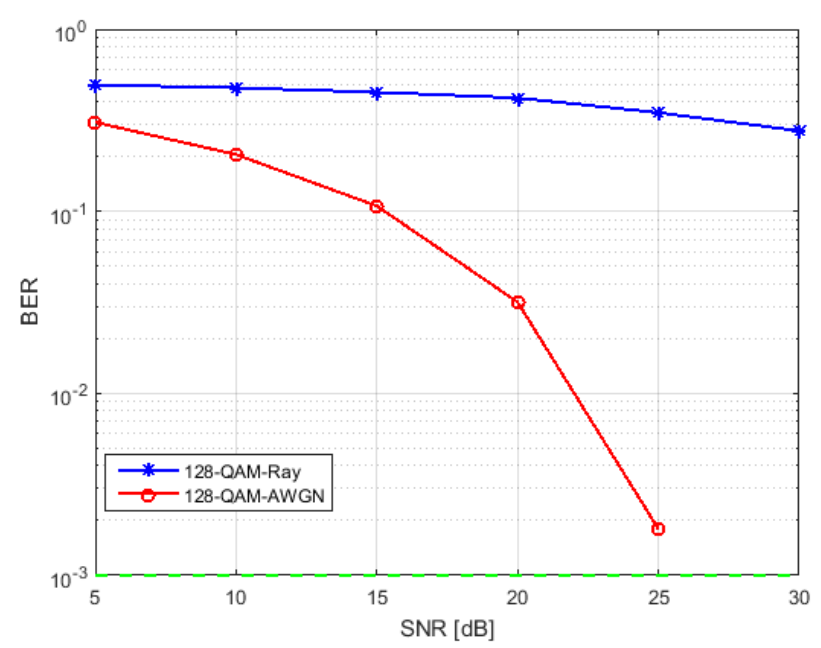

Fig. 10. Relation between BER and SNR for 128 QAM over AWGN and Rayleigh.

gained BER and offered SNR is digitally processed for variant modulation schemes (QAM and PSK). Hence, the overall system performance in terms of the BER and SNR is presented for both the conventional modulation techniques as the BPSK, QPSK and the advanced configurations of modulation like the 128 and 256 QAM over the AWGN channel.

In addition, the simulation results show that, whenever, the received power of the signal is increased the BER is decreased until achieve the accepted criteria of tested modulation scheme.

As is seen in Fig. 13, the channel model can play a big role in degrading the system performance for both the SNR and BER, thus, the necessity for adapting the required power of the receiver at agreed limit of error is appeared. Hence, the required SNR to achieve a good reception with a modulation format over the AWGN is much lower in compare with the signal passes through a noisy mode like the Rayleigh channel model.

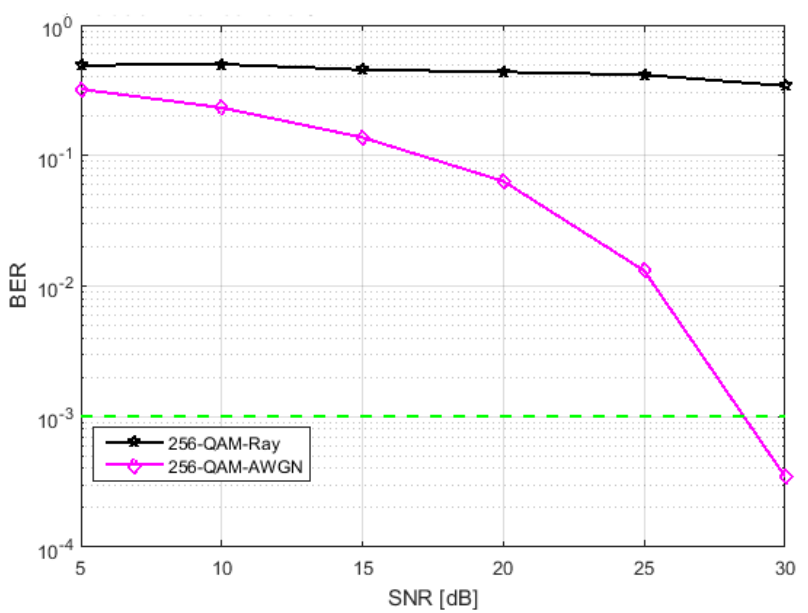

Fig. 11. Relation between BER and SNR for 256 QAM over AWGN and Rayleigh. 


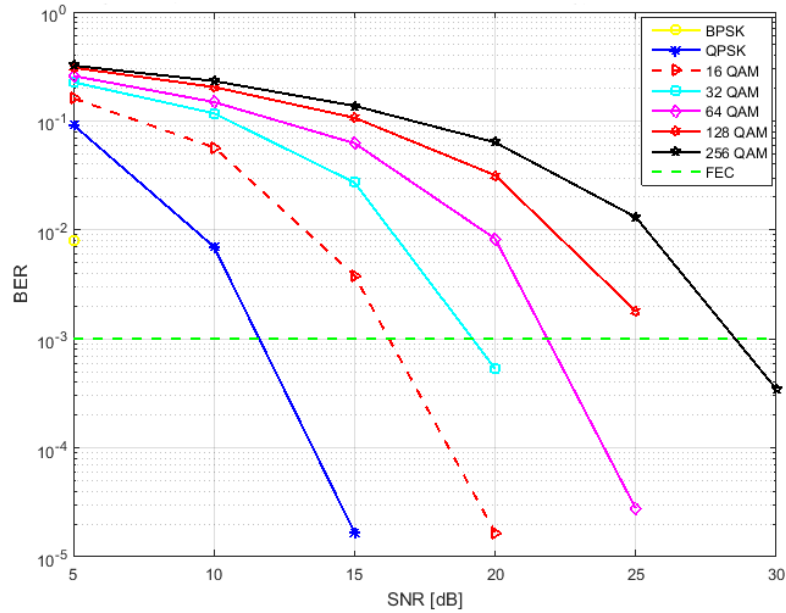

Fig. 12. System performance in terms of BER and SNR for most common modulation formats including both 128 and 256 QAM over AWGN channel.

This essentially, because of the induced channel response that directly impacts the key parameters of the transmitted signal. Hence, the phase and amplitude of the sample are changed according to the type of channel response.

As a result, to provide a high data rate transmission along with reliable communication system the trade-off relation between modulation schemes and received power is adjusted precisely over the Rayleigh channel.

It's also shown in both Fig. 12, and Fig. 13, that the minimum limits of the SNR that improve the BER to $10^{-3}$ totally differ between transmission over the AWGN than Rayleigh channel. Hence, the modulation formats with the LoS channel can offer a better performance (BER, SNR) compared to the NLoS channel.

Besides, the sharply decreased BER is achieved by increasing the SNR levels in both Rayleigh and AWGN channels

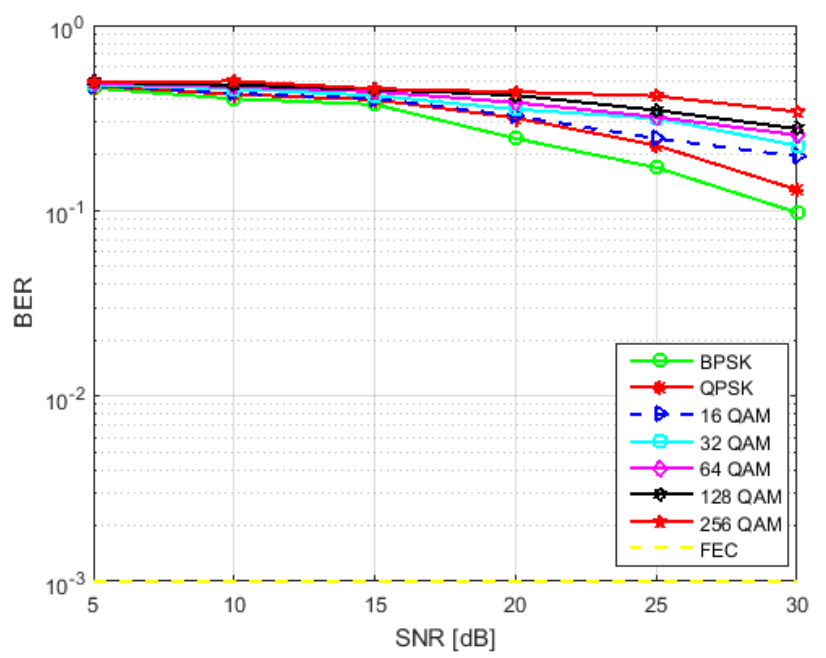

Fig. 13. System performance in terms of BER and SNR for most common modulation formats including both 128 and 256 QAM over Rayleigh channel. considering that BER is higher with the Rayleigh than the normal AWGN.

From these considered scenarios, it's found that the dynamic range of received power per each transmitted signal depends on modulation format type and utilized channel model.

It's worth noting that, among these 7 investigated modulation formats, the BPSK can be applied with a lower power requirement, while the 256 QAM requires a higher level of power due to the increment of bits number for each transmitted sample. Hence, for those applications which prefer power than the BW efficiency, the low order modulation format can be a more suitable solution than the higher modulation schemes and vice versa. Thus, the modulation techniques are employed depending on the type of applied application.

As such, with the future generation of mobile, the higher modulation schemes are very recommended due to the increased need for a higher bit rate.

This, however, comes up with some technical difficulties such as large-power reception which must be supplied by the conventional big cells to recover the transmitted signal with the higher modulation formats.

To solve such a challenge, nowadays technology, like the small cells can be a good solution for the modern generation of mobile networks which basically aims to provide huge amounts of data efficiently [22].

To test the proposed system, a MATLAB simulation is utilized to check whether the transmission operation with the proposed modulation formats over different channel models is achieved correctly or not.

In this testing process, the constellation table is considered to demonstrate the behaviour of electrical back-to-back transmission system toward the digital signal. Hence, depending on the recognizable receiving of the constellates, the reception can be confirmed, and the level of a suitable receiver power is decided.

The constellation map decision is changed, based on key factors like type of modulation technique and employed channel model.

Accordingly, the required SNR is adapted to treat any probable distortion of the received signal.

As it is mentioned before, the number of probable positions on the constellation map is calculated according to equation (12).

The experimental work shows the examined results for different cases of the constellation table under the most common kinds of modulation formats including the higher order as the 128 , and 256 QAM and in presence of variant types of noise like the AWGN and Rayleigh.

As it is clear in Fig. 14, using the phase attribute, the probability of points appearance from transmitting 1 bit over the applied system is only two. These points which basically organized in two options are achieved at the accepted limit of the BER with SNR equivalents to 7 and $58 \mathrm{~dB}$ over the AWGN and Rayleigh channels respectively. 


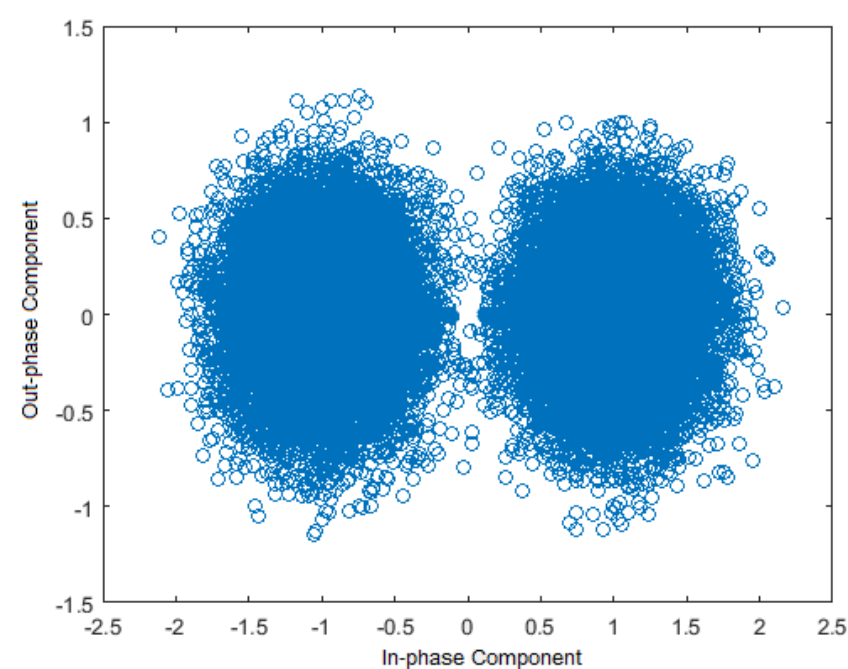

Fig. 14. BPSK modulation format of receiver.

The increased number of bits ( 2 bits), as is shown in Fig. 15, leads to raising the probability of achieving extra positions at the constellation table to four. Hence, depending on the phase feature, the organized constellations can reflect the accuracy of transmission over channels models. Thus, at a BER equal to $10^{-3}$ the constellation table gives four sorted positions with SNR level variant between $12 \mathrm{~dB}$ for the AWGN and $63 \mathrm{~dB}$ with the Rayleigh.

In addition, as is seen in Fig. 16, whenever the number of bits is enlarged (4 bits), the appearance chances of receiving new complex numbers are increased resulting in decreasing the distances between the adjacent constellations. Thus, utilizing both phase and amplitude, 16 opportunities are offered in a sorted way at the map, referring that received signal is accepted at the required limit. The recorded SNR for a good reception with 16 QAM and over the AWGN is $16.5 \mathrm{~dB}$ while a higher ratio is accounted to the Rayleigh at $67.5 \mathrm{~dB}$.

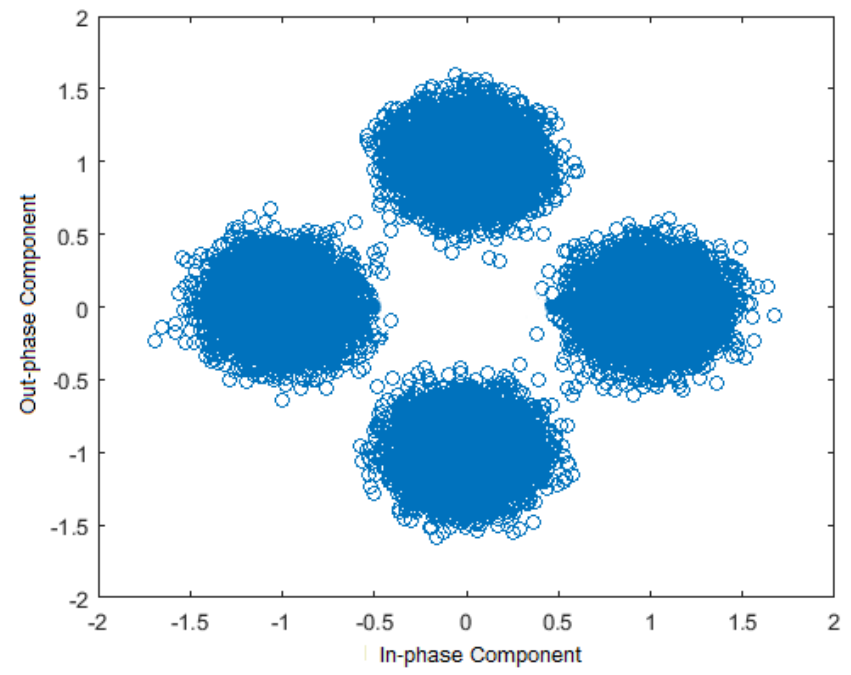

Fig. 15. QPSK modulation format of receiver.

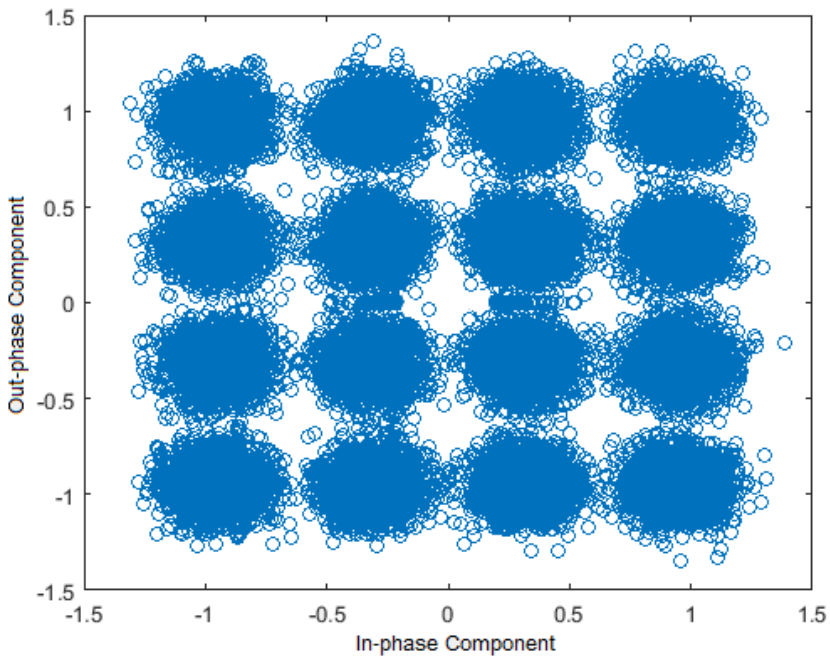

Fig. 16. 16 QAM modulation format of receiver.

Besides, as it is noticed in Fig. 17, employing 5 bits for each sample leads to constructing the 32 QAM modulation, which also depends on both phase and amplitude to accommodate the expanded cases of complex numbers at the constellation table. Thus, 32 different cases are received announcing that reached signal is correctly positioned with the gained BER. The agreeable limits of errors are obtained with diverse SNR levels, where $19.5 \mathrm{~dB}$ is assigned for the AWGN and $70.5 \mathrm{~dB}$ for the Rayleigh.

Moreover, as it is clear in Fig. 18, by raising the number of bits to six, the currently utilized modulation format, 64 QAM is achieved. Thus, employing the aspects of phase and magnitude, 64 arranged shapes are drawn at the constellation map proving that digital signal is received optimally. The registered values of the obtained SNR for this arranged reception over the AWGN and Rayleigh channels are $22.5 \mathrm{~dB}$ and $73.5 \mathrm{~dB}$ respectively.

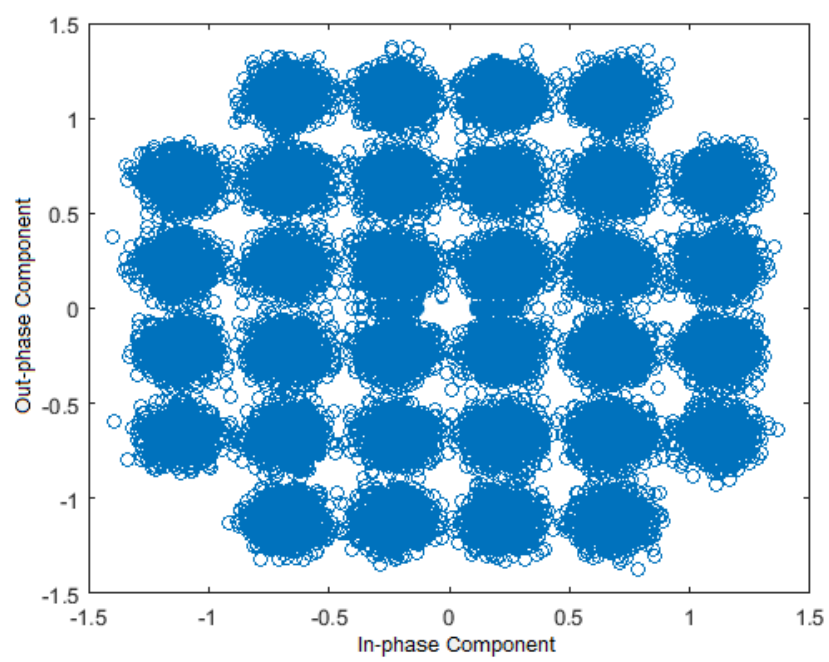

Fig. 17. 32 QAM modulation format of receiver. 


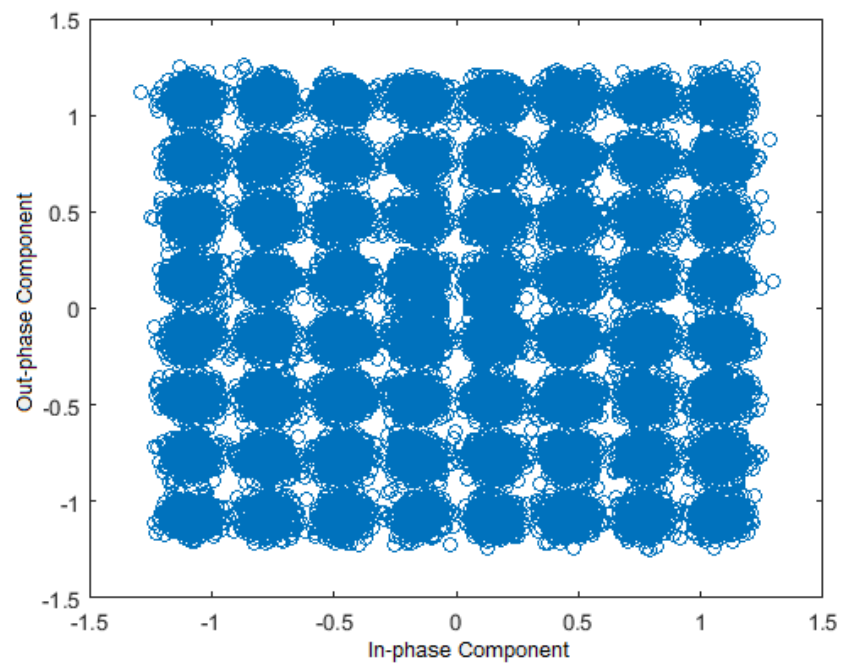

Fig. 18. 64 QAM modulation format of receiver.

Regarding the newly proposed higher order modulation formats, firstly, as it is shown in Fig. 19, seven bits are specified for each transmitted sample to shape a new coordinated constellation table with 128 available options for representing the transmitted data.

Based on the phase and amplitude utilities, the correctly accommodated samples indicate that complex numbers of the transmitted signal are received successfully. The gained levels of the SNR declare that the AWGN needs to about $25.5 \mathrm{~dB}$ while the Rayleigh comes up with $76.5 \mathrm{~dB}$ to get the acceptable limit of the error's ratio.

Eventually, as it is seen in Fig. 20, eight bits are decided for every transmitted sample introducing an extra number of the selections reach to 256 obtainable codes.

The harmonic reception of the transmitted samples demonstrates that the receiver side is working effectively. Hence, depending on the correct obtaining of both phase and magnitude

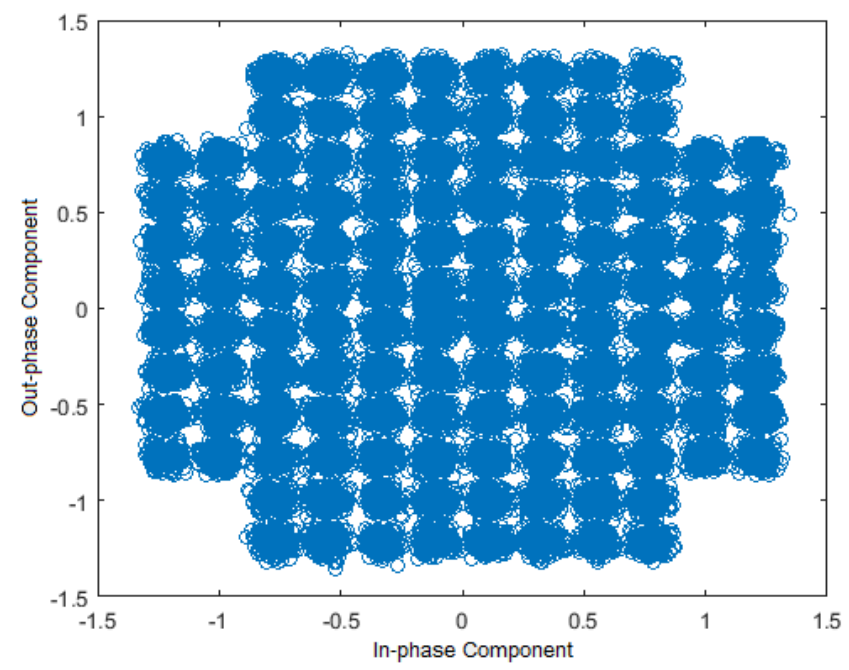

Fig. 19. 128 QAM modulation format of receiver.

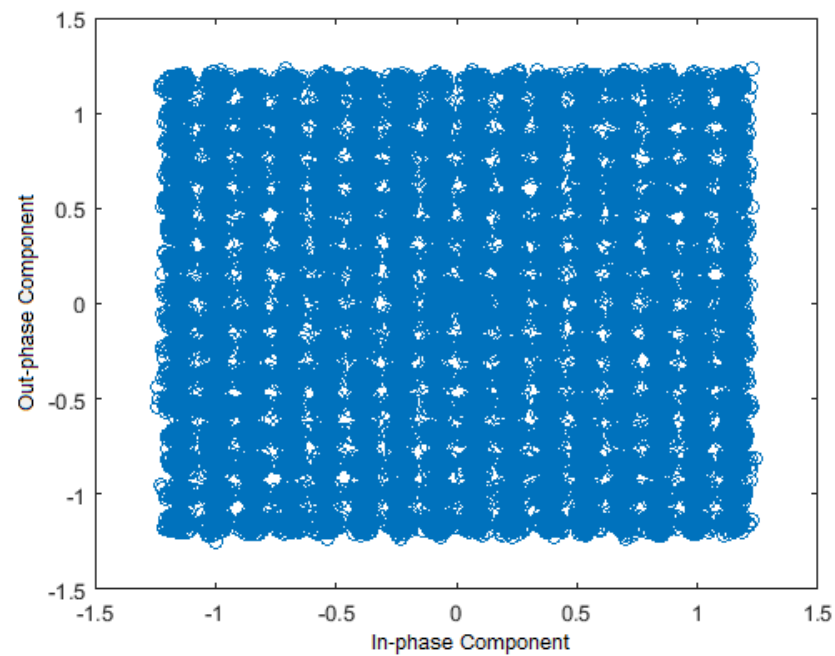

Fig. 20. 256 QAM modulation format of receiver.

at the 256 QAM constellation map, the standard limit of errors is accepted.

Higher levels of the computed SNR are assigned with varied models of channels where $29 \mathrm{~dB}$ is accounted to the AWGN and around $80 \mathrm{~dB}$ is consumed with the Rayleigh.

\section{CONCLUSION}

In this study, new configurations for the PHY of future mobile technology are provided by considering higher approaches of modulation than the currently applied in the telecommunication systems. More specifically, advanced modulation formats like 128 QAM and 256 QAM employing the current waveform (OFDM) is proposed, investigated and evaluated. The performance analysis of the relatively higher schemes of modulation are theoretically and experimentally assessed under different channel conditions (AWGN and Rayleigh). The experimental work shows that higher modulation schemes can supply more data bit rate but less transmission reliability due to the increased rate of errors compared to lower modulation formats. In addition, it reveals that, for all examined modulation formats, the achieved level of the error rate is minimized with the AWGN while maximized with Rayleigh noise channel. Moreover, the findings clear that the higher order modulations are more suitable for purposes that need great BW efficiency but down power efficiency, while lower order modulations are more recommended for purposes that include less power and error but lower BW efficiency. Thus, the promoted modulation schemes can improve the transmission channel capacity of a future wireless mobile system in comparison with the currently applied format (64 QAM). However, the effect of adding an extra bit for each transmitted sample is directly related to the raised requirement of power for each received complex number due to increased interference among transmitted points in the constellation table. Hence, increased levels of the BER synchronize with the enlarged schemes of modulation. Thus, the gained values of the SNR reflect the close relationship between increased bit rate and required receiving power. As results, the higher modulation formats are not preferred alone for the future generation of mobile networks, since the new 
predicated scenarios expected higher channel capacities with lower levels of power. Consequently, sophisticated technology like the small cell is highly recommended, with the high-order modulation formats, due to their efficient ability in increasing the coverage of transmission for future wireless networks of mobile. The achieved results are evaluated mathematically and physically utilizing a MATLAB simulation code.

\section{REFERENCES}

[1] Y. U. E. Xiao, H. Haas, and S. Member, "Index Modulation Techniques for Next-Generation Wireless Networks," vol. 5, pp. 16693-16746, 2017.

[2] M. R. Kadhum, T. Kanakis, A. Al-sherbaz, and R. Crockett, "Digital Chunk Processing with Orthogonal GFDM Doubles Wireless Channel Capacity," Advances in Intelligent Systems and Computing, vol 857, pp. 719-731, Nov. 2018. doi:https://doi.org/10.1007/978-3-030-01177-2-53.

[3] I. Chandran and K. A. Reddy, "Comparative analysis of various Channel Estimations under different Modulation Schemes," vol. 1, no. 3, pp. 832-837, 2017.

[4] K. A. Reddy, "Evaluation of BER for AWGN, Rayleigh Fading Channels under M-QAM Modulation Scheme," pp. 3081-3086, 2016.

[5] M. R. Kadhum, T. Kanakis, and R. Crockett, "Dynamic Bit Loading with the OGFDM Waveform Maximises Bit-Rate of Future Mobile Communications," Advances in Intelligent Systems and Computing, Proceedings of computing conference 2019, London, 2019.

[6] M. Barnela, "Digital Modulation Schemes Employed in Wireless Communication: A Literature review," vol. 2, no. 2, pp. 15-21, 2014.

[7] M. R. Kadhum, T. Kanakis, and R. Crockett, "Intra-Channel Interference Avoidance with the OGFDM Boosts Channel Capacity of Future Wireless Mobile Communication," Advances in Intelligent Systems and Computing, Proceedings of computing conference 2019, London, 2019.

[8] C. U. Ndujiuba, O. Oni, and A. E. Ibhaze, "Comparative Analysis of Digital Modulation Techniques in LTE 4G Systems," vol. 5, no. 2, pp. 60-66, 2015.

[9] W. Jin et al., "Improved Performance Robustness of DSP-Enabled Flexible ROADMs Free from Optical Filters and O-E-O Conversions," IEEE J. Opt. Commun. Netw., vol. 8, no. 8, p. 521, 2016.
[10] T. Hwang, C. Yang, S. Member, G. Wu, S. Li, and G. Y. Li, "OFDM and Its Wireless Applications: A Survey," vol. 58, no. 4, pp. 1673-1694, 2009.

[11] M. R. Kadhum, "New Multi-Carrier Candidate Waveform for the $5 G$ Physical Layer of Wireless Mobile Networks," IEEE, Proceedings of Wireless Days 2019, Manchester, 2019.

[12] N. Sood, "BER Performance of OFDM-BPSK and -QPSK over Nakagami-m Fading Channels," no. 1, pp. 88-90, 2010.

[13] G. H. Im, D. D. Harman, G. Huang, A. V. Mandzik, M. H. Nguyen, and J. J. Werner, "51.84 Mb/s 16-CAP ATM LAN Standard" IEEE J. Sel. Areas Commun., vol. 13, no. 4, pp. 620-632, 1995.

[14] G. Song and L. Cuthbert, "Physical Layer Simulations of the Broadband Wireless LANs Based on OWSS Signalling Technique," pp. 442-445, 2010.

[15] R. Yang, J. Bian, and C. Zhu, "Fixed point Dual Circular 32-QAM performance for Wireless USB," 2011 Int. Conf. Consum. Electron. Commun. Networks, CECNet 2011 - Proc., pp. 1310-1314, 2011.

[16] K. Nagarajan, V. V. Kumar, and S. Sophia, "Analysis of OFDM Systems for High Bandwidth Application," pp. 168-171, 2017.

[17] M. M. Madankar and P. S. Ashtankar, "Performance analysis of BPSK modulation scheme for different channel conditions," no. 2002, 2016.

[18] S. W. Smith, Digital signal processing. 1999.

[19] Q. Yang, S. Chen, Y. Ma, and W. Shieh, "Real-time reception of multigigabit coherent optical OFDM signals," vol. 17, no. 10, pp. 873-879, 2009.

[20] M. Ghogho, D. McLernon, E. Alameda-Hernandez, and A. Swami, "Channel estimation and symbol detection for block transmission using data-dependent superimposed training," IEEE Signal Process. Lett., vol. 12, no. 3, pp. 226-229, 2005.

[21] Z. Karaelmas and Ü. Zonguldak, "Performances of M-PSK and M-QAM Modulated OFDM Signals over AWGN and Rayleigh Fading Channels," pp. 523-526, 2010.

[22] H. Haboobi, M. R. Kadhum, and A. Al-sherbaz, "Utilise Higher Modulation Formats with Heterogeneous Mobile Networks Increases Wireless Channel Transmission," Advances in Intelligent Systems and Computing, Proceedings of computing conference 2019, London, 2019. 